\title{
ON SOLVABILITY OF MIXED MONOTONE OPERATOR EQUATIONS WITH APPLICATIONS TO MIXED QUASIMONOTONE DIFFERENTIAL SYSTEMS INVOLVING DISCONTINUITIES ${ }^{1}$
}

\author{
S. HEIKKILÄ and M. KUMPULAINEN \\ University of Oulu \\ Department of Mathematics \\ SF-90570 Oulu 57, FINLAND \\ V. LAKSHMIKANTHAM \\ Florida Institute of Technology \\ Department of Applied Mathematics \\ Melbourne, Florida 32901-6988, USA
}

\begin{abstract}
In this paper we shall first study solvability of mixed monotone systems of operator equations in an ordered normed space by using a generalized iteration method. The obtained results are then applied to prove existence of coupled extremal quasisolutions of the systems of first and second order mixed quasimonotone differential equations with discontinuous right hand sides. Most of the results deal with systems in a Banach space ordered by a regular order cone.
\end{abstract}

Key words: Operator equations, systems of differential equations in ordered Banach spaces, discontinuous nonlinearity, mixed quasimonotonicity, generalized iteration method.

AMS (MOS) subject classifications: $\quad 47 \mathrm{H} 15,34 \mathrm{~B} 15,34 \mathrm{G} 20$.

\section{INTRODUCTION}

In [6] existence of coupled minimal and maximal quasisolutions of the system

$$
u_{i}^{\prime}(t)=f_{i}\left(t, u_{i}(t),[u]_{p_{i}}(t),[u]_{q_{i}}(t)\right), t \in[0, T], u_{i}(0)=u_{0_{i}}, i=1, \ldots, n
$$

is studied by assuming that the functions $f_{i}$ are continuous, mixed quasimonotone and satisfy one-sided Lipschitz condition with respect to $u_{i}$. The last assumption is replaced in [5] by $g_{i}$ monotonicity of the functions $f_{i}$.

In this paper we shall extend the above mentioned results to the case when the functions $f_{i}$ are allowed to be discontinuous in all of their variables. These extensions will be

\footnotetext{
${ }^{1}$ Received: July, 1991. Revised: September, 1991.
} 
obtained as applications of a result proved first for operator equations of mixed monotone type in an ordered normed space. This result will be applied also to the second order mixed quasimonotone system

$$
\begin{gathered}
u_{i}^{\prime \prime}(t)=f_{i}\left(t, u_{i}(t),[u]_{p_{i}}(t),[u]_{q_{i}}, u_{i}^{\prime}(t)\right), t \in[0, T] \\
u_{i}(0)=u_{0_{i}}, u_{i}^{\prime}(0)=u_{1_{i}}
\end{gathered}
$$

in an ordered Banach space.

\section{ON MIXED MONOTONE OPERATOR EQUATIONS}

In this section we shall study solvability of the operator equations

$$
v=A(v, w), w=A(w, v)
$$

in an ordered normed space $X$.

Theorem 2.1: $\quad$ Given a subset $Z$ of $X$ possessing the least and the greatest element, and a mapping $A: Z \times Z \rightarrow Z$, assume that

(A1) $A(\cdot, z)$ is increasing and $A(z, \cdot)$ is decreasing for each $z \in Z$.

(A2) $\left(A\left(v_{j}, w_{j}\right)\right)_{j=1}^{\infty}$ converges in $Z$ whenever $\left(v_{j}\right)_{j=1}^{\infty}$ and $\left(w_{j}\right)_{j=1}^{\infty}$ are sequences in $Z$, one being increasing and the other decreasing.

Then the system (2.1) has a solution $(v, w)$ such that

(i) $\quad v \leq w$ and $\bar{v}, \bar{w} \in[v, w]$ for any other solution $(\bar{v}, \bar{w})$ of (2.1).

Proof: Note first that $X \times X$, equipped with componentwise addition and scalar multiplication, is an ordered normed space with respect to the norm and the partial ordering given by

$$
\|(v, w)\|=\|v\|+\|w\| \text { and }(v, w) \leq(\bar{v}, \bar{w}) \text { if and only if } v \leq \bar{v} \text { and } \bar{w} \leq w .
$$

Denoting $Y=Z \times Z$ it follows from $(A 1)$ that the equation

$$
F(v, w)=(A(v, w), A(w, v))
$$

defines an increasing mapping $F: Y \rightarrow Y$. If $\left(u_{j}\right)_{j=1}^{\infty}=\left(\left(v_{j}, w_{j}\right)\right)_{j=1}^{\infty}$ is an increasing sequence in $Y$, then $\left(v_{j}\right)_{j=1}^{\infty}$ is increasing and $\left(w_{j}\right)_{j=1}^{\infty}$ decreasing in $Z$. The hypothesis $(A 2)$ implies that the sequences $\left(A\left(v_{j}, w_{j}\right)\right)_{j=1}^{\infty}$ and $\left(A\left(w_{j}, v_{j}\right)\right)_{j=1}^{\infty}$ converge in $Z$. Thus, by $(a)$ and $(b)$, the sequence $\left(F u_{j}\right)_{j=1}^{\infty}$ converges in $Y$.

The above proof shows that the mapping $F: Y \rightarrow Y$ given by $(b)$ is increasing and right compact. Moreover, since $v_{0}=\min Z$ and $w_{0}=\max Z$ exist, then $a=\left(v_{0}, w_{0}\right)$ is the least 
element of $Y$. Thus, $F$ has by Theorem 3.1 of [2] the least fixed point $u=(v, w)$ in $Y$. Moreover, $u=\sup \left\{F u_{\alpha} \mid \alpha \in \Lambda\right\}$, where $\left(u_{\alpha}\right)_{\alpha \in \Lambda}$ is the longest transfinite sequence, indexed by ordinals, which satisfies

$$
u_{0}=a, \text { and if } 0<\alpha \in \Lambda \text { then } u_{\alpha}=s u p_{\beta<\alpha} F u_{\beta} \text { and } u_{\alpha}<F u_{\alpha} \text {. }
$$

The above results imply by $(a)$ and $(b)$ that $(v, w)$ is a solution of $(2.1)$, and that $v=\sup \left\{A\left(v_{\alpha}, w_{\alpha}\right) \mid \alpha \in \Lambda\right\}$ and $w=\inf \left\{A\left(w_{\alpha}, v_{\alpha}\right) \mid \alpha \in \Lambda\right\}$, where $\left(v_{\alpha}\right)_{\alpha \in \Lambda}$ and $\left(w_{\alpha}\right)_{\alpha \in \Lambda}$ are the longest transfinite sequences which satisfy

(I) If $0<\alpha \in \Lambda$ then $v_{\alpha}=\sup _{\beta<\alpha} A\left(v_{\beta}, w_{\beta}\right), w_{\alpha}=i n f_{\beta<\alpha} A\left(w_{\beta}, v_{\beta}\right)$, and the inequalities $v_{\alpha} \leq A\left(v_{\alpha}, w_{\alpha}\right), A\left(w_{\alpha}, v_{\alpha}\right) \leq w_{\alpha}$ hold, at least one of them being strict.

If $(\bar{v}, \bar{w})$ is a solution of (2.1), it follows from $(2.1)$ and $(b)$ that $(\bar{v}, \bar{w})$ is a fixed point of $F$ in $Y$. Because $(v, w)$ is the least one, then $(v, w) \leq(\bar{v}, \bar{w})$, i.e. $v \leq \bar{v}$ and $\bar{w} \leq w$. In particular, we can choose $\bar{v}=w$ and $\bar{w}=v$, whence $v \leq w$. Since $(\bar{w}, \bar{v})$ is also a solution of (2.1), it follows that $\bar{v}, \bar{w} \in[v, w]$, so that (i) holds.

Remark 2.1: $\quad$ If $A$ in Theorem 2.1 is continuous, then also $F$ is continuous, whence the sequence $\left(u_{\alpha}\right)_{\alpha \in \Lambda}$ is reduced to a finite or infinite sequence of ordinary iterations $F^{j} a, j \in \mathbb{N}$. In particular, $v=\lim _{j \rightarrow \infty} v_{j}$ and $w=\lim _{j \rightarrow \infty} w_{j}$, where $\left(v_{j}\right)_{j=0}^{\infty}$ and $\left(w_{j}\right)_{j=0}^{\infty}$ are defined by

$$
v_{j+1}=A\left(v_{j}, w_{j}\right), w_{j+1}=A\left(w_{j}, v_{j}\right), j \in \mathrm{N}
$$

\section{APPLICATIONS TO FIRST ORDER DIFFERENTIAL SYSTEMS}

Given an ordered Banach space $E$ and $J=[0, T]$, consider the differential system

$$
u_{i}^{\prime}(t)=f_{i}\left(t, u_{i}(t),[u]_{p_{i}}(t),[u]_{q_{i}}(t)\right) \text { for a.e. } t \in J, u_{i}(0)=u_{0_{i}}, i=1, \ldots, n,
$$

where $f_{i}: J \times E^{n} \rightarrow E$, and $p_{i}, q_{i} \in \mathbb{N}, i=1, \ldots, n$ satisfy $p_{i}+q_{i}=n-1$. In the notation $u=\left(u_{i},[u]_{p_{i}},[u]_{q_{i}}\right.$ ) for $u \in E^{n}$ the term $[u]_{p_{i}}$ is formed by $p_{i}$ coordinates of $u$, different from $u_{i}$, and $[u]_{q_{i}}$ contains the remaining ones.

The absolutely continuous and a.e. differentiable functions $v=\left(v_{1}, \ldots, v_{n}\right)$ and $w=\left(w_{1}, \ldots, w_{n}\right)$ from $J$ to $E^{n}$ are called coupled lower and upper quasisolutions of (3.1), if

and

$$
v_{i}^{\prime}(t) \leq f_{i}\left(t, v_{i}(t),[v]_{p_{i}}(t),[w]_{q_{i}}(t)\right) \text { for a.e. } t \in J, v_{i}(0) \leq u_{0_{i}}, i=1, \ldots, n
$$




$$
w_{i}^{\prime}(t) \geq f_{i}\left(t, w_{i}(t),[w]_{p_{i}}(t),[v]_{q_{i}}(t)\right) \text { for a.e. } t \in J, w_{i}(0) \geq u_{0_{i}}, i=1, \ldots, n .
$$

If the equalities hold in (3.2) and in (3.3), we say that $v, w$ are coupled quasisolutions of (3.1). If $v=w=u$, we say that $u$ is a lower, an upper or an ordinary solution of (3.1), respectively. In the following, we shall equip $C\left(J, E^{n}\right)$ with the maximum norm and pointwise ordering, and

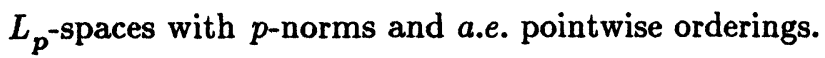

\section{1. g-monotone and mixed quasimonotone real systems.}

Consider first the case when $E=\mathbb{R}$. Given $f_{i}: J \times \mathbb{R}^{n} \rightarrow \mathbb{R}$ and $u_{0_{i}} \in \mathbb{R}, i=1, \ldots, n$, assume that

(f1) (3.1) has coupled lower and upper quasisolutions $v_{0}, w_{0}$ such that $v_{0} \leq w_{0}$.

(f2) $f_{i}(\cdot, u(\cdot))$ is Lebesgue measurable on $J$ and whenever $u \in\left[v_{0}, w_{0}\right]$ is absolutely continuous.

(f3) $f_{i}\left(t, u_{i}[u]_{p_{i}},[u]_{q_{i}}\right)$ is increasing with respect to $[u]_{p_{i}}$ on $\left[\left[v_{0}\right]_{p_{i}}(t),\left[w_{0}\right]_{p_{i}}(t)\right]$ and decreasing with respect to $\left[u_{q_{i}}\right]$ on $\left[\left[v_{0}\right]_{q_{i}}(t),\left[w_{0}\right]_{q_{i}}(t)\right]$ for all $u_{i} \in\left[v_{0_{i}}(t), w_{0_{i}}(t)\right]$ and for a.e. $t \in J$.

(f4) There is a Borel measurable function $g_{i} \in L_{\infty}\left(\mathbb{R}, \mathbb{R}_{+}\right)$with positive essential infimum such that $u_{i} \mapsto \frac{f_{i}\left(t, u_{1}, \ldots, u_{i}, \ldots, u_{n}\right)}{g_{i}\left(u_{i}\right)}$ is increasing in $\left[v_{0_{i}}(t), w_{0_{i}}(t)\right]$ for all $u=\left(u_{1}, \ldots, u_{n}\right) \in\left[v_{0}(t), w_{0}(t)\right]$ and for a.e. $t \in J$.

Theorem 3.1: If the hypotheses $(f 1)-(f 4)$ hold for each $i=1, \ldots, n$, then (3.1) has coupled quasisolutions $v, w$ satisfying $v_{0} \leq v \leq w \leq w_{0}$, such that $\bar{v}, \bar{w} \in[v, w]$ whenever $\bar{v}$, $\bar{w} \in\left[v_{0}, w_{0}\right]$ are coupled quasisolutions of (3.1).

Proof: Denote $Z=\left\{z \in\left[v_{0}, w_{0}\right] \mid z\right.$ is absolutely continuous $\}$, and let $i \in\{1, \ldots, n\}$ be given. The hypotheses $(f 1)-(f 4)$ imply that for all $v, w \in Z$

$$
\int_{0}^{t} \frac{f_{i}\left(x, v_{i}(x),[v]_{p_{i}}(x),[w]_{q_{i}}(x)\right)}{g_{i}\left(v_{i}(x)\right)} d x \leq \int_{0}^{t} \frac{f_{i}\left(x, w_{0_{i}}(x),\left[w_{0}\right]_{p_{i}}(x),\left[v_{0}\right]_{q_{i}}(x)\right)}{g_{i}\left(w_{0_{i}}(x)\right)} d x, t \in J .
$$

From (3.3) it follows (see also [7], V. 38.3) that

$$
\int_{0}^{t} \frac{f_{i}\left(x, w_{0_{i}}(x),\left[w_{0}\right]_{p_{i}}(x),\left[v_{0}\right]_{q_{i}}(x)\right)}{g_{i}\left(w_{0_{i}}(x)\right)} d x \leq \int_{0}^{t} \frac{w_{0_{i}}^{\prime}(x)}{g_{i}\left(w_{0_{i}}(x)\right)} d x \leq \int_{u_{0_{i}}}^{w_{0_{i}}(t)} \frac{d y}{g_{i}(y)}, t \in J .
$$

The reverse inequalities hold when $w_{0_{i}}$ is replaced by $v_{0_{i}}$ and vice versa.

Thus, the equation 


$$
\int_{u_{0_{i}}}^{A_{i}(v, w)(t)} \frac{d y}{g_{i}(y)}=\int_{0}^{t} \frac{f_{i}\left(x, v_{i}(x),[v]_{p_{i}}(x),[w]_{q_{i}}(x)\right)}{g_{i}\left(v_{i}(x)\right)} d x, t \in J
$$

defines a mapping $A_{i}: Z \times Z \rightarrow C(J, \mathbb{R})$ such that $v_{0_{i}} \leq A_{i}(v, w) \leq w_{0_{i}}$. From $(a)$ it follows that $A_{i}(v, w)$ is absolutely continuous on $J$ for each $(v, w) \in Z \times Z$. The hypotheses $(f 3)$ and (f4) ensure that $A_{i}(\cdot, z)$ is increasing and $A_{i}(z, \cdot)$ is decreasing for each $z \in Z$.

The above considerations hold for each $i=1, \ldots, n$, so that the equations $(a)$ define a mapping $A=\left(A_{1}, \ldots, A_{n}\right): Z \times Z \rightarrow Z$ which satisfies condition $(A 1)$ of Theorem 2.1. To prove that condition $(A 2)$ holds, let $\left(v_{j}\right)_{j=1}^{\infty}$ be an increasing and $\left(w_{j}\right)_{j=1}^{\infty}$ a decreasing sequence in $Z$. For given $i \in\{1, \ldots, n\}$ the functions

$$
h_{j}^{i}(x)=\frac{f_{i}\left(x, v_{j_{i}}(x),\left[v_{j}\right]_{p_{i}}(x),\left[w_{j}\right]_{q_{i}}(x)\right)}{g_{i}\left(v_{j_{i}}(x)\right)}, x \in J
$$

form an increasing sequence $\left(h_{j}^{i}\right)_{j=1}^{\infty}$ in $L^{1}(J, \mathbb{R})$, bounded above by a function

$$
h_{0}^{i}(x)=\frac{f_{i}\left(x, w_{0_{i}}(x),\left[w_{0}\right]_{p_{i}}(x),\left[v_{0}\right]_{q_{i}}(x)\right)}{g_{i}\left(w_{0_{i}}(x)\right)}, x \in J .
$$

Thus, the sequence $\left(h_{j}^{i}\right)_{j=1}^{\infty}$ converges by the Monotone Convergence Theorem to a function $h^{i} \in L_{1}(J, \mathbb{R})$, and

Defining $u_{i}: J \rightarrow \mathbb{R}$ by

$$
\lim _{j \rightarrow \infty} \int_{0}^{t} h_{j}^{i}(x) d x=\int_{0}^{t} h^{i}(x) d x, t \in J
$$

$$
\int_{u_{0_{i}}}^{u_{i}(t)} \frac{d y}{g_{i}(y)}=\int_{0}^{t} h^{i}(x) d x, t \in J
$$

and denoting $M_{i}=$ ess sup $g_{i}$, it follows from $(a),(b)$ and $(d)$ that

$$
0 \leq \frac{1}{M_{i}}\left(u_{i}(t)-A_{i}\left(v_{j}, w_{j}\right)(t)\right) \leq \int_{A_{i}\left(v_{j}, w_{j}\right)(t)}^{u_{i}(t)} \frac{d y}{g_{i}(y)} \leq \int_{0}^{T}\left(h^{i}(x)-h_{j}^{i}(x)\right) d x, t \in J .
$$

This and $(c)$ imply that the sequence $\left(A_{i}\left(v_{j}, w_{j}\right)\right)_{j=1}^{\infty}$ converges uniformly on $J$ to $u_{i}$. Thus, denoting $u=\left(u_{1}, \ldots, u_{n}\right)$, then $A\left(v_{j}, w_{j}\right) \rightarrow u$ in $X=C\left(J, \mathbb{R}^{n}\right)$ with respect to the uniform norm. Moreover, it is easy to see that $u \in Z$, whence $\left(A\left(v_{j}, w_{j}\right)\right)_{j=1}^{\infty}$ converges in $Z$. This convergence can be proved similarly also in the case $\left(v_{j}\right)_{j=1}^{\infty}$ is a decreasing and $\left(w_{j}\right)_{j=1}^{\infty}$ an increasing sequence in $Z$. Thus, $A$ satisfies the condition $(A 2)$ of Theorem 2.1. Noting also that $v_{0}$ is the least and $w_{0}$ is the greatest element of $Z$, it follows from Theorem 2.1 that (2.1) has a solution $(v, w)$ such that $(i)$ holds. 
Denoting $v=\left(v_{1}, \ldots, v_{n}\right)$, and $w=\left(w_{1}, \ldots, w_{n}\right)$, it follows from (2.1) that $v_{i}=A_{i}(v, w)$ for each $i=1, \ldots, n$. Thus, $(a)$ is equivalent to

$$
\int_{u_{0_{i}}}^{v_{i}(t)} \frac{d y}{g_{i}(y)}=\int_{0}^{t} \frac{v_{i}^{\prime}(x) d x}{g_{i}\left(v_{i}(x)\right)}=\int_{0}^{t} \frac{f_{i}\left(x, v_{i}(x),[v]_{p_{i}}(x),[w]_{q_{i}}(x)\right)}{g_{i}\left(v_{i}(x)\right)} d x, t \in J .
$$

This is in turn equivalent to

$$
\frac{v_{i}^{\prime}(t)}{g_{i}\left(v_{i}(t)\right)}=\frac{f_{i}\left(t, v_{i}(t),[v]_{p_{i}}(t),[w]_{q_{i}}(t)\right)}{g_{i}\left(v_{i}(t)\right)}, \text { for a.e. } t \in J, v_{i}(0)=u_{0_{i}},
$$

and hence to

$$
v_{i}^{\prime}(t)=f_{i}\left(t, v_{i}(t),[v]_{p_{i}}(t),[w]_{q_{i}}(t)\right) \text { for a.e. } t \in J, v_{i}(0)=u_{0_{i}}, i=1, \ldots, n .
$$

Similarly, it can be shown that

$$
w_{i}^{\prime}(t)=f_{i}\left(t, w_{i}(t),[w]_{p_{i}}(t),[v]_{q_{i}}(t)\right) \text { for a.e. } t \in J, w_{i}(0)=u_{0_{i}}, i=1, \ldots, n .
$$

Thus, $v$ and $w$ are coupled quasisolutions of (3.1) and $v_{0} \leq v \leq w \leq w_{0}$.

If $\bar{v}, \bar{w}$ are coupled quasisolutions of $(3.1)$ in $\left[v_{0}, w_{0}\right]$, then they belong to $Z$, whence the above equivalences imply that $(\bar{v}, \bar{w})$ is a solution of (2.1) when the components of $A$ are defined by $(a)$. This and the result $(i)$ of Theorem 2.1 imply that $\bar{v}, \bar{w} \in[v, w]$.

As a consequence of Theorem 3.1 we obtain,

Proposition 3.1: Given the functions $g_{i} \in L_{\infty}\left(\mathbb{R}, \mathbb{R}_{+}\right)$and $h_{i}: J \times \mathbb{R}^{n} \rightarrow \mathbb{R}, i=1, \ldots, n$, assume that for each $i=1, \ldots, n, g_{i}$ has positive essential infimum, that $h_{i}$ is Borel measurable, that $h_{i}(t, \cdot)$ is increasing on $\mathbb{R}^{n}$ for a.e. $t \in J$, and that there is $m_{i} \in L_{1}\left(J, \mathbb{R}_{+}\right)$such that

$$
\left|h_{i}(t, u)\right| \leq m_{i}(t) \text { for all } u \in \mathbb{R}^{n} \text { and for a.e. } t \in J \text {. }
$$

Then the system

$$
u_{i}^{\prime}(t)=g_{i}\left(u_{i}(t)\right) h_{i}(t, u(t)) \text { for a.e. } t \in J, u_{i}(0)=u_{0_{i}}, i=1, \ldots, n
$$

has for each $\left(u_{0_{1}}, \ldots, u_{0_{n}}\right) \in \mathbb{R}_{+}^{n}$ the least and the greatest solution.

Proof: The assumptions given for $g_{i}$ and condition (3.4) imply that the equations

$$
\int_{u_{0_{i}}}^{w_{0_{i}}(t)} \frac{d y}{g_{i}(y)}=\int_{0}^{t} m_{i}(x) d x, \int_{u_{0_{i}}}^{v_{0_{i}}(t)} \frac{d y}{g_{i}(y)}=-\int_{0}^{t} m_{i}(x) d x
$$

define an upper solution $w_{0}=\left(w_{0_{1}}, \ldots, w_{0_{n}}\right)$ and a lower solution $v_{0}=\left(v_{0_{1}}, \ldots, v_{0_{n}}\right)$ of the system (3.5). Moreover, it is easy to see that the functions $f_{i}=g_{i} h_{i}$ satisfy the conditions 
$(f 2)-(f 4)$ when $p_{i}=n-1$ and $q_{i}=0, i=1, \ldots, n$. (Borel measurability of $g_{i}$ is not needed since $\frac{f_{i}}{g_{i}}=h_{i}$. As for the proof of $(f 2)$ see [8]). Thus, Theorem 3.1, applied to this special case, implies that the system (3.5) has the least solution $v$ and the greatest solution $w$ between $v_{0}$ and $w_{0}$.

If $u=\left(u_{1}, \ldots, u_{n}\right)$ is any solution of $(3.5)$, then its components are absolutely continuous in $J$. Thus, it follows from (3.5) that

$$
\int_{u_{0_{i}}}^{u_{i}(t)} \frac{d y}{g_{i}(y)}=\int_{0}^{t} h_{i}(x, u(x)) d x, t \in J, i=1, \ldots, n .
$$

From (3.4), (a) and (b) we obtain

$$
v_{0_{i}}(t) \leq u_{i}(t) \leq w_{0_{i}}(t) \text { for all } t \in J .
$$

Thus, $v_{0} \leq u \leq w_{0}$, so that $v$ is the least and $w$ is the greatest of all the solutions of (3.5).

\subsection{Mixed quasimonotone systems in an ordered Banach space.}

Next we shall give an extension of Theorem 1.4.1 of [6] to the case when $f=\left(f_{1}, \ldots, f_{n}\right)$ is a mapping from $J \times E^{n}$ to $E^{n}$, where $E$ is an ordered Banach space, by allowing $f$ also to possess discontinuities.

Theorem 3.2: $\quad$ Let $E$ be an ordered Banach space with regular order cone, and let $f_{i}: J \times E^{n} \rightarrow E$ and $u_{0_{i}} \in E, i=1, \ldots, n$ satisfy the conditions $(f 1),(f 3)$,

(f5) $f_{i}(\cdot, u(\cdot))$ is strongly measurable whenever $u: J \rightarrow E^{n}$ is absolutely continuous and a.e. differentiable, and

(f6) there is $\mu_{i} \in L_{1}\left(J, \mathbb{R}_{+}\right)$such that $u_{i} \mapsto f_{i}\left(t, u_{1}, \ldots, u_{i}, \ldots, u_{n}\right)+\mu_{i}(t) u_{i}$ is increasing on $\left[v_{0_{i}}(t), w_{0_{i}}(t)\right]$ for all $u=\left(u_{1}, \ldots, u_{n}\right) \in\left[v_{0}(t), w_{0}(t)\right]$ and for a.e. $t \in J$.

Then the results of Theorem 3.1 hold.

Proof: Denote $Z=\left\{z \in\left[v_{0}, w_{0}\right] \mid z\right.$ is absolutely continuous and a.e. differentiable\}. The given hypotheses imply that the equation

$$
\lambda_{i}(v, w)(t)=f_{i}\left(t, v_{i}(t),[v]_{p_{i}}(t),[w]_{q_{i}}(t)\right)+\mu_{i}(t) v_{i}(t), t \in J
$$

defines for each $i=1, \ldots, n$ a mapping $\lambda_{i}: Z \times Z \rightarrow L_{1}(J, E)$, which is increasing in the first argument and decreasing in the second one. Thus, the initial value problem

$$
u_{i}^{\prime}(t)+\mu_{i}(t) u_{i}(t)=\lambda_{i}(v, w)(t) \text { for a.e. } t \in J, u_{i}(0)=u_{0_{i}}
$$


has for each $i=1, \ldots, n$ and $v, w \in Z$ a unique absolutely continuous solution $u_{i}=A_{i}(v, w)$ given by

$$
A_{i}(v, w)(t)=\exp \left(-\int_{0}^{t} \mu_{i}(x) d x\right)\left(u_{0_{i}}+\int_{0}^{t} \exp \left(\int_{0}^{x} \mu_{i}(s) d s\right) \lambda_{i}(v, w)(x) d x\right), t \in J .
$$

The so obtained functions $A_{i}: Z \times Z \rightarrow C(J, E)$ have absolutely continuous and a.e. differentiable values, and they possess the mixed monotonicity conditions stated for the functions $\lambda_{i}$ above. Moreover, from (3.2), (a) and (b) it follows when $v=v_{0}$ and $w=w_{0}$ that $r_{i}=u_{i}-v_{0_{i}}$ satisfies for each $i=1, \ldots, n$

$$
r_{i}^{\prime}(t)+\mu_{i}(t) r_{i}(t) \geq 0 \text { for a.e. } t \in J, r_{i}(0) \geq 0
$$

which implies that

$$
r_{i}(t) \geq r_{i}(0) \exp \left(-\int_{0}^{t} \mu_{i}(x) d x\right) \geq 0 \text { for each } t \in J .
$$

Thus, $v_{0_{i}} \leq A_{i}\left(v_{0}, w_{0}\right), i=1, \ldots, n$. By the analogous reasoning it can be shown that $A_{i}\left(w_{0}, v_{0}\right) \leq w_{0}, i=1, \ldots, n$.

The above results imply that $A=\left(A_{1}, \ldots, A_{n}\right)$ is a mapping from $Z \times Z$ to $Z$, and satisfies condition $(A 1)$ of Theorem 2.1 .

Assume now that $\left(v_{j}\right)_{j=1}^{\infty}$ is an increasing and $\left(w_{j}\right)_{j=1}^{\infty}$ a decreasing sequence in $Z$. The sequences $\left(\lambda_{i}\left(v_{j}, w_{j}\right)(t)\right)_{j=1}^{\infty}, t \in J$ are increasing and are contained in the order interval $\left[\lambda_{i}\left(v_{0}, w_{0}\right)(t), \lambda_{i}\left(w_{0}, v_{0}\right)(t)\right]$, whence they converge, because the order cone $K$ of $E$ is regular. Denote

$$
h^{i}(t)=\lim _{j \rightarrow \infty} \lambda_{i}\left(v_{j}, w_{j}\right)(t), t \in J .
$$

Since $K$ is also normal, there is a positive constant $c$ such that

$$
\left\|\lambda_{i}\left(v_{j}, w_{j}\right)(t)\right\| \leq(1+c)\left(\left\|\lambda_{i}\left(v_{0}, w_{0}\right)(t)\right\|+\left\|\lambda_{i}\left(w_{0}, v_{0}\right)(t)\right\|\right) \text { for a.e. } t \in J .
$$

Thus, $h^{i} \in L_{1}(J, E)$ by the Dominated Convergence Theorem for Bochner Integrals (cf. [4]), and

Defining $\nu_{i}: J \rightarrow E$ by

$$
\lim _{j \rightarrow \infty} \int_{0}^{t} \lambda_{i}\left(v_{j}, w_{j}\right)(x) d x=\int_{0}^{t} h^{i}(x) d x, t \in J
$$

$$
\nu_{i}(t)=\exp \left(-\int_{0}^{t} \mu_{i}(x) d x\right)\left(u_{0_{i}}+\int_{0}^{t} \exp \left(\int_{0}^{x} \mu_{i}(s) d s\right) h^{i}(x) d x\right), t \in J
$$

it follows from $(c),(d)$ and $(e)$ that

$$
\lim _{j \rightarrow \infty} A_{i}\left(v_{j}, w_{j}\right)(t)=\nu_{i}(t) t \in J .
$$


Moreover,

$$
0 \leq \nu_{i}(t)-A_{i}\left(v_{j}, w_{j}\right)(t) \leq \exp \left(\int_{0}^{T} \mu_{i}(x) d s\right) \int_{0}^{T}\left(h^{i}(x)-\lambda_{i}\left(v_{j}, w_{j}\right)(x)\right) d x, t \in J,
$$

which implies by $(d)$ and the normality of $K$ that the convergence in $(f)$ is uniform on $J$. Thus, denoting $\nu=\left(\nu_{1}, \ldots, \nu_{n}\right)$, then $A\left(v_{j}, w_{j}\right) \rightarrow \nu$ in $C\left(J, E^{n}\right)$. Obviously, $\nu \in Z$, whence $\left(A\left(v_{j}, w_{j}\right)\right)_{j=1}^{\infty}$ converges in $Z$. This convergence in the case when $\left(v_{j}\right)_{j=1}^{\infty}$ is decreasing and $\left(w_{j}\right)_{j=1}^{\infty}$ is increasing is proved similarly. Thus, $A$ satisfies the condition (A2) of Theorem 2.1 , whence $(2.1)$ has a solution $(v, w)$ such that $(i)$ holds.

Denoting $v=\left(v_{1}, \ldots, v_{n}\right)$, and $w=\left(w_{1}, \ldots, w_{n}\right)$, it follows from $(2.1)$ and $(c)$ that

$$
v_{i}(t)=\exp \left(-\int_{0}^{t} \mu_{i}(x) d x\right)\left(u_{0_{i}}+\int_{0}^{t} \exp \left(\int_{0}^{x} \mu_{i}(s) d s\right) \lambda_{i}(v, w)(x) d x\right), t \in J .
$$

This is easily seen to be equivalent to

$$
v_{i}^{\prime}(t)+\mu_{i}(t) v_{i}(t)=\lambda_{i}(v, w)(t) \text { for a.e. } t \in J, v_{i}(0)=u_{0_{i}},
$$

and hence to

$$
v_{i}^{\prime}(t)=f_{i}\left(t, v_{i}(t),[v]_{p_{i}}(t),[w]_{q_{i}}(t)\right) \text { for a.e. } t \in J, v_{i}(0)=u_{0_{i}}, i=1, \ldots, n .
$$

Similarly, it can be shown that

$$
w_{i}^{\prime}(t)=f_{i}\left(t, w_{i}(t),[w]_{p_{i}}(t),[v]_{q_{i}}(t)\right) \text { for a.e. } t \in J, w_{i}(0)=u_{0_{i}}, i=1, \ldots, n \text {. }
$$

Thus, $v$ and $w$ are coupled quasisolutions of (3.1) and $v_{0} \leq v \leq w \leq w_{0}$.

If $\bar{v}, \bar{w}$ are coupled quasisolutions of (3.1) in $\left[v_{0}, w_{0}\right]$, then they belong to $Z$, whence the above equivalences imply that $(\bar{v}, \bar{w})$ is a solution of $(2.1)$ when the components of $A$ are defined by $(c)$. Thus, the result $(i)$ of Theorem 2.1 ensures that $\bar{v}, \bar{w} \in[v, w]$.

Lemma 3.1: The hypothesis $(f 1)$ holds in Theorem 3.2 for all fixed $u_{0_{1}}, \ldots, u_{0_{n}} \in E$, if for each $i=1, \ldots, n$ there exist $a_{i}^{j} \in L_{1}(J, \mathbb{R})$ and $b_{i}^{j} \in L_{1}(J, E), j=1,2$ such that

$$
a_{i}^{1}(t) u_{i}+b_{i}^{1}(t) \leq f_{i}(t, u) \leq a_{i}^{2}(t) u_{i}+b_{i}^{2}(t) \text { for a.e. } t \in J \text { and for all } u \in E^{n}
$$

Proof: Let $u_{0_{1}}, \ldots, u_{0_{n}} \in E$ be given. Direct calculations show that the equations

$$
v_{0_{i}}(t)=\exp \left(\int_{0}^{t} a_{i}^{1}(x) d x\right)\left(u_{0_{i}}+\int_{0}^{t} \exp \left(-\int_{0}^{x} a_{i}^{1}(s) d s\right) b_{i}^{1}(x) d x\right), t \in J, i=1, \ldots, n
$$

and 


$$
w_{0_{i}}(t)=\exp \left(\int_{0}^{t} a_{i}^{2}(x) d x\right)\left(u_{0_{i}}+\int_{0}^{t} \exp \left(-\int_{0}^{x} a_{i}^{2}(s) d s\right) b_{i}^{2}(x) d x\right), t \in J, i=1, \ldots, n
$$

define coupled lower and upper quasisolutions $v_{0}=\left(v_{0_{1}}, \ldots, v_{0_{n}}\right)$ and $w_{0}=\left(w_{0_{1}}, \ldots, w_{0_{n}}\right)$ of the system (3.1) such that $v_{0} \leq w_{0}$.

In the special case when $p_{i}=n-1$ and $q_{i}=0, i=1, \ldots, n$ we obtain from Theorem 3.2 the following result for the system

$$
u_{i}^{\prime}(t)=f_{i}(t, u(t)) \text { for a.e. } t \in J, u_{i}(0)=u_{0_{i}}, i=1, \ldots, n,
$$

which generalizes Theorems 3.1 and 3.2 of [1].

Proposition 3.2: Given an ordered Banach space $E$ with regular order cone, assume that the functions $f_{i}: J \times E^{n} \rightarrow E$ satisfy the hypotheses of Lemma 3.1, that $f_{i}(\cdot, u(\cdot))$ is strongly measurable whenever $u: J \rightarrow E^{n}$ is absolutely continuous and a.e. differentiable, and that there is $\mu_{i} \in L_{1}\left(J, \mathbb{R}_{+}\right)$such that $u \mapsto f_{i}(t, u)+\mu_{i}(t) u_{i}$ is increasing for a.e. $t \in J$ and for each $i=1, \ldots, n$. Then the system (3.9) has for each choice of $u_{0_{1}}, \ldots, u_{0_{n}} \in E$ the least and the greatest solution.

Proof: Given $u_{0_{1}}, \ldots, u_{0_{n}} \in E$, it is easy to see that hypotheses of Theorem 3.2 hold with $v_{0}, w_{0}$ defined by $(3.7)-(3.8)$. Thus, the system (3.9) has the least solution $v$ and the greatest solution $w$ between $v_{0}$ and $w_{0}$.

If $u=\left(u_{1}, \ldots, u_{n}\right)$ is any solution of (3.9), it follows from (3.6), (3.7) and (3.9) that $r_{i}=u_{i}-v_{0_{i}}$ satisfies for each $i=1, \ldots, n$

$$
r_{i}^{\prime}(t)-a_{i}^{1}(t) r_{i}(t) \geq 0 \text { for a.e. } t \in J, r_{i}(0)=0,
$$

which implies that

$$
r_{i}(t) \geq r_{i}(0) \exp \left(\int_{0}^{t} a_{i}^{1}(x) d x\right)=0 \text { for each } t \in J .
$$

Thus, $v_{0} \leq u$. Similarly it can be shown that $u \leq w_{0}$, whence all the solutions of (3.9) are between $v_{0}$ and $w_{0}$. Consequently, $v$ is the least and $w$ the greatest of all the solutions of the system (3.9).

Remarks 3.1: Condition $(f 2)$ holds, for instance when $f_{i}$ is a "standard function" in the sense defined in [8], and in particular, when $f_{i}$ is Borel measurable. If each $h_{i}$ in Proposition 3.1 is standard, then the assumption on the Borel measurability of $h_{i}$ is not needed.

Condition $(f 5)$ of Theorem 3.2 holds, if $E$ is separable and each $f_{i}$ is a standard 
function (cf. [8]).

If in Theorems 3.1 and 3.2 the functions $g_{i}$ are continuous and each $f_{i}$ is a Carathéodory function, it follows from Remark 2.1 that the asserted coupled quasisolutions $v$ and $w$ of (3.1) are obtained as the uniform limits of the successive approximations given by (2.2). Moreover, if the functions $f_{i}$ and $\mu_{i}$ are continuous then $v, w$ are coupled extremal quasisolutions of the system (1) given in Introduction. Similar special cases are obtained also for Propositions 3.1 and 3.2.

Theorem 3 in [9] contains the special case of Theorem 3.2 where $E=\mathbb{R}$ and the functions $f_{i}$ are mixed monotone, i.e. each $\mu_{i}$ is the zero function in the condition $(f 6)$.

\section{APPLICATIONS TO SECOND ORDER DIFFERENTIAL SYSTEMS}

Given an ordered Banach space $E$, consider the differential system

$$
\begin{gathered}
u_{i}^{\prime \prime}(t)=f_{i}\left(t, u_{i}(t),[u]_{p_{i}}(t),[u]_{q_{i}}(t), u_{i}^{\prime}(t)\right) \text { for a.e. } t \in J, \\
u_{i}(0)=u_{0_{i}}, u_{i}^{\prime}(0)=u_{1_{i}}, i=1, \ldots, n,
\end{gathered}
$$

where $f_{i}: J \times E^{n+1} \rightarrow E, i=1, \ldots, n$. The functions $v=\left(v_{1}, \ldots, v_{n}\right)$ and $w=\left(w_{1}, \ldots, w_{n}\right): J \rightarrow E^{n}$ are said to be coupled lower and upper quasisolutions of (4.1) if their components possess absolutely continuous and a.e. differentiable first derivatives, and if

$$
\begin{gathered}
v_{i}^{\prime \prime}(t) \leq f_{i}\left(t, v_{i}(t),[v]_{p_{i}}(t),[w]_{q_{i}}(t), v_{i}^{\prime}(t)\right) \text { for a.e. } t \in J \\
v_{i}(0) \leq u_{0_{i}}, v_{i}^{\prime}(0) \leq u_{1_{i}}, i=1, \ldots, n
\end{gathered}
$$

and

$$
\begin{gathered}
w_{i}^{\prime \prime}(t) \geq f_{i}\left(t, w_{i}(t),[w]_{p_{i}}(t),[v]_{q_{i}}(t), w_{i}^{\prime}(t)\right) \text { for a.e. } t \in J, \\
w_{i}(0) \geq u_{0_{i}}, w_{i}^{\prime}(0) \geq u_{1_{i}}, i=1, \ldots, n .
\end{gathered}
$$

If the equalities hold in (4.2) and in (4.3), we say that $v, w$ are coupled quasisolutions of (4.1). In particular, if $v=w=u$, we say that $u$ is a lower, an upper or an ordinary solution of (4.1), respectively.

Our considerations are based on Theorem 2.1 and the following Lemma, which is a consequence of Example 2.1 and Lemma 6.1 of [3].

Lemma 4.1: $\quad$ If $\mu \in L_{1}(J, \mathbb{R})$ and $C \in L_{1}(J, E)$ then the IVP

$$
u^{\prime \prime}(t)+\mu(t) u^{\prime}(t)=C(t) \text { for a.e. } t \in J, u(0)=u_{0}, u^{\prime}(0)=u_{1}
$$


has for each choice of $u_{0}, u_{1} \in E$ the unique solution

$$
u(t)=u_{0}+\int_{0}^{t} \exp \left(-\int_{0}^{x} \mu(y) d y\right) u_{1} d x+\int_{0}^{t} \int_{0}^{x} \exp \left(\int_{x}^{y} \mu(s) d s\right) C(y) d y d x .
$$

Moreover, if $v, w$ are lower and upper solutions of (4.4) then $v \leq u \leq w$ and $v^{\prime} \leq u^{\prime} \leq w^{\prime}$.

Assume now that the functions $f_{i}: J \times E^{n+1} \rightarrow E$ satisfy for each $i=1, \ldots, n$

( $f a)$ (4.1) has coupled lower and upper quasisolutions $v_{0}, w_{0}$, such that $v_{0} \leq w_{0}$ and $v_{0}^{\prime} \leq w_{0}^{\prime}$.

(fb) $f_{i}(\cdot, u(\cdot), v(\cdot))$ is strongly measurable on $J$ whenever $u \in\left[v_{0}, w_{0}\right], v \in\left[v_{0_{i}}^{\prime}, w_{0_{i}}^{\prime}\right]$, and $u^{\prime}$ and $v$ are absolutely continuous and a.e. differentiable.

(fc) $f_{i}\left(t, u_{i},[u]_{p_{i}},[u]_{q_{i}}, v\right)$ is increasing with respect to $u_{i}$ on $\left[v_{0_{i}}(t), w_{0_{i}}(t)\right]$ and to $[u]_{p_{i}}$ on $\left[\left[v_{0}\right]_{p_{i}}(t),\left[w_{0}\right]_{p_{i}}(t)\right]$, and decreasing with respect to $[u]_{q_{i}}$ on $\left[\left[v_{0}\right]_{q_{i}}(t),\left[w_{0}\right]_{q_{i}}(t)\right]$ for all $v \in\left[v_{0_{i}}^{\prime}(t), w_{0_{i}}^{\prime}(t)\right]$ and for a.e. $t \in J$.

(fd) There is $\mu_{i} \in L_{1}\left(J, \mathbb{R}_{+}\right)$such that $v \mapsto f_{i}(t, u, v)+\mu_{i}(t) v$ is increasing on $\left[v_{0_{i}}^{\prime}(t), w_{0_{i}}^{\prime}(t)\right]$ for all $u \in\left[v_{0}(t), w_{0}(t)\right]$ and for a.e. $t \in J$.

Theorem 4.1: $\quad$ Let $E$ be an ordered Banach space with regular order cone $K$. If the functions $f_{i}: J \times E^{n+1} \rightarrow E$ satisfy the hypotheses $(f a)-(f d)$, then the system (4.1) has coupled quasisolutions $v, w$ satisfying $\left(v_{0}, v_{0}^{\prime}\right) \leq\left(v, v^{\prime}\right) \leq\left(w, w^{\prime}\right) \leq\left(w_{0}, w_{0}^{\prime}\right)$, such that $\bar{v}$, $\bar{w} \in[v, w]$ and $\bar{v}^{\prime}, \bar{w}^{\prime} \in\left[v^{\prime}, w^{\prime}\right]$ whenever $\bar{v}, \bar{w} \in\left[v_{0}, w_{0}\right]$ are coupled quasisolutions of (4.1) such that $\bar{v}^{\prime}, \bar{w}^{\prime} \in\left[v_{0}^{\prime}, w_{0}^{\prime}\right]$.

Proof: Denote $Z=\left\{u \in\left[v_{0}, w_{0}\right] \mid u^{\prime}\right.$ is absolutely continuous a.e. differentiable and $\left.u^{\prime} \in\left[v_{0}^{\prime}, w_{0}^{\prime}\right]\right\}$, and define a norm and a partial ordering in $X=C_{1}\left(J, E^{n}\right)$ by

$$
\|u\|=\sup \left\{\|u(t)\|+\left\|u^{\prime}(t)\right\| \mid t \in J\right\}
$$

and

$$
u \leq v \text { if and only if } u(t) \leq v(t) \text { and } u^{\prime}(t) \leq v^{\prime}(t) \text { for all } t \in J .
$$

The given hypotheses imply that the equation

$$
C_{i}(v, w)(t)=f_{i}\left(t, v_{i}(t),[v]_{p_{i}}(t),[w]_{q_{i}}(t), v_{i}^{\prime}(t)\right)+\mu_{i}(t) v_{i}^{\prime}(t), t \in J
$$

defines for each $i=1, \ldots, n$ a mapping $C_{i}: Z \times Z \rightarrow L_{1}(J, E)$, which is increasing in the first argument and decreasing in the second one. Thus, the initial value problem

$$
u_{i}^{\prime \prime}(t)+\mu_{i}(t) u_{i}^{\prime}(t)=C_{i}(v, w)(t) \text { for a.e. } t \in J, u_{i}(0)=u_{0_{i}}, u_{i}^{\prime}(0)=u_{1_{i}}
$$

has by Lemma 4.1 for each $i=1, \ldots, n$ and $v, w \in Z$ a unique absolutely continuous solution, 
$u_{i}=A_{i}(v, w)$, given by

$$
A_{i}(v, w)(t)=u_{0_{i}}+\int_{0}^{t} \exp \left(-\int_{0}^{x} \mu_{i}(y) d y\right) u_{1_{i}} d x+\int_{0}^{t} \int_{0}^{x} \exp \left(\int_{x}^{y} \mu_{i}(s) d s\right) C_{i}(v, w)(y) d y d x .
$$

The so obtained functions $A_{i}(v, w): J \rightarrow E$ have absolutely continuous and a.e. differentiable first derivatives, and they possess the mixed monotonicity conditions stated for the functions $C_{i}$ above. Moreover, from (4.2), (a) and (b) it follows when $v=v_{0}$ and $w=w_{0}$ that $r=v_{0_{i}}-u_{i}$ is for each $i=1, \ldots, n$ a lower solution of

$$
r^{\prime \prime}(t)+\mu_{i}(t) r^{\prime}(t)=0 \text { for a.e. } t \in J, r(0)=r^{\prime}(0)=0 .
$$

Since the zero function is the only solution of this $I V P$, it follows from Lemma 4.1 that $v_{0_{i}} \leq A_{i}\left(v_{0}, w_{0}\right)$ and $v_{0_{i}}^{\prime} \leq A_{i}\left(v_{0}, w_{0}\right)^{\prime}$. Similarly, it follows from Lemma 4.1 that $A_{i}\left(w_{0}, v_{0}\right) \leq w_{0_{i}}$ and $A_{i}\left(w_{0}, v_{0}\right)^{\prime} \leq w_{0_{i}}^{\prime}$ for each $i=1, \ldots, n$.

The above results imply that $A=\left(A_{1}, \ldots, A_{n}\right)$ is a mapping from $Z \times Z$ to $Z$, and satisfies condition $(A 1)$ of Theorem 2.1 .

Assume now that $\left(v_{j}\right)_{j=1}^{\infty}$ is an increasing and $\left(w_{j}\right)_{j=1}^{\infty}$ a decreasing sequence in $Z$. The sequences $\left(C_{i}\left(v_{j}, w_{j}\right)(t)\right)_{j=1}^{\infty}, t \in J$ are increasing and are contained in the order interval $\left[C_{i}\left(v_{0}, w_{0}\right)(t), C_{i}\left(w_{0}, v_{0}\right)(t)\right]$, whence they converge, because the order cone $K$ of $E$ is regular. Denote

$$
h^{i}(t)=\lim _{j \rightarrow \infty} C_{i}\left(v_{j}, w_{j}\right)(t), t \in J .
$$

Since $K$ is also normal, there is a positive constant $c$ such that

$$
\left\|C_{i}\left(v_{j}, w_{j}\right)(t)\right\| \leq(1+c)\left(\left\|C_{i}\left(v_{0}, w_{0}\right)(t)\right\|+\left\|C_{i}\left(w_{0}, v_{0}\right)(t)\right\|\right) .
$$

Thus, $h^{i} \in L_{1}(J, E)$ by Dominated Convergence Theorem for Bochner integrals and

Defining $\nu_{i}: J \rightarrow E$ by

$$
\lim _{j \rightarrow \infty} \int_{0}^{t} C_{i}\left(v_{j}, w_{j}\right)(x) d x=\int_{0}^{t} h^{i}(x) d x, t \in J .
$$

then

$$
\nu_{i}(t)=u_{0_{i}}+\int_{0}^{t} \exp \left(-\int_{0}^{x} \mu_{i}(y) d y\right) u_{1_{i}} d x+\int_{0}^{t} \int_{0}^{x} \exp \left(\int_{x}^{y} \mu_{i}(s) d s\right) h^{i}(y) d y d x, t \in J
$$

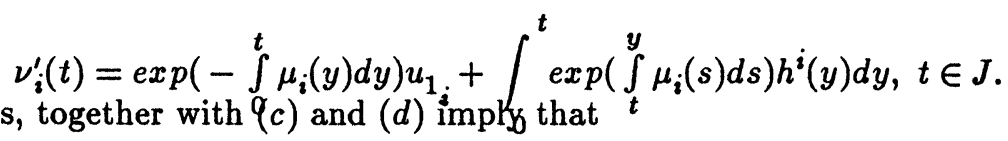

$$
\lim _{j \rightarrow \infty} A_{i}\left(v_{j}, w_{j}\right)(t)=\nu_{i}(t), \lim _{j \rightarrow \infty} A_{i}\left(v_{j}, w_{j}\right)^{\prime}(t)=\nu_{i}^{\prime}(t) t \in J .
$$


Moreover,

and

$$
0 \leq \nu_{i}(t)-A_{i}\left(v_{j}, w_{j}\right)(t) \leq T \exp \left(\int_{0}^{T} \mu_{i}(x) d x\right) \int_{0}^{T}\left(h^{i}(x)-C_{i}\left(v_{j}, w_{j}\right)(x)\right) d x, t \in J
$$

$$
0 \leq \nu_{i}^{\prime}(t)-A_{i}\left(v_{j}, w_{j}\right)^{\prime}(t) \leq \exp \left(\int_{0}^{T} \mu_{i}(x) d x\right) \int_{0}^{T}\left(h^{i}(x)-C_{i}\left(v_{j}, w_{j}\right)(x)\right) d x, t \in J
$$

These inequalities, $(d)$ and the normality of $K$ imply that the convergence in $(e)$ is uniform on $J$. Thus, denoting $\nu=\left(\nu_{1}, \ldots, \nu_{n}\right)$, then $A\left(v_{j}, w_{j}\right) \rightarrow \nu$ in $C_{1}\left(J, E^{n}\right)$. Obviously, $\nu \in Z$, whence $\left(A\left(v_{j}, w_{j}\right)\right)_{j=1}^{\infty}$ converges in $Z$. This convergence in the case when $\left(v_{j}\right)_{j=1}^{\infty}$ is decreasing and $\left(w_{j}\right)_{j=1}^{\infty}$ is increasing is proved similarly. Thus, $A$ satisfies the condition (A2) of Theorem 2.1 , whence $(2.1)$ has a solution $(v, w)$ such that $(i)$ holds.

Denoting $v=\left(v_{1}, \ldots, v_{n}\right)$, and $w=\left(w_{1}, \ldots, w_{n}\right)$, it follows from (2.1) and $(c)$ that for each $i=1, \ldots, n$

$$
v_{i}(t)=u_{0_{i}}+\int_{0}^{t} \exp \left(-\int_{0}^{x} \mu_{i}(y) d y\right) u_{1_{i}} d x+\int_{0}^{t} \int_{0}^{x} \exp \left(\int_{x}^{y} \mu_{i}(s) d s\right) C_{i}(v, w)(y) d y d x .
$$

By Lemma 4.1 this holds if and only if

$$
v_{i}^{\prime \prime}(t)+\mu_{i}(t) v_{i}^{\prime}(t)=C_{i}(v, w)(t) \text { for a.e. } t \in J, v_{i}(0)=u_{0_{i}}, v_{i}^{\prime}(0)=u_{1_{i}}
$$

or equivalently,

$$
\begin{gathered}
v_{i}^{\prime \prime}(t)=f_{i}\left(t, v_{i}(t),[v]_{p_{i}}(t),[w]_{q_{i}}(t), v_{i}^{\prime}(t)\right) \text { for a.e. } t \in J \\
v_{i}(0)=u_{0_{i}}, v_{i}^{\prime}(0)=u_{1_{i}}
\end{gathered}
$$

for each $i=1, \ldots, n$. Similarly, it can be shown that

$$
\begin{gathered}
w_{i}^{\prime \prime}(t)=f_{i}\left(t, w_{i}(t),[w]_{p_{i}}(t),[v]_{q_{i}}(t), w_{i}^{\prime}(t)\right) \text { for a.e. } t \in J \\
w_{i}(0)=u_{0_{i}}, w_{i}^{\prime}(0)=u_{1_{i}}
\end{gathered}
$$

for each $i=1, \ldots, n$. Thus, $v$ and $w$ are coupled quasisolutions of (4.1) in $Z$. The above equivalences imply in turn that such coupled quasisolutions of (4.1) form also a solution of (2.1) with the components of $A$ given by $(c)$. From the definitions of $Z$ and the partial ordering in $X=C_{1}\left(J, E^{n}\right)$ and from the result $(i)$ in Theorem 2.1, it then follows that $\left(v_{0}, v_{0}^{\prime}\right) \leq\left(v, v^{\prime}\right) \leq\left(w, w^{\prime}\right) \leq\left(w_{0}, w_{0}^{\prime}\right), \bar{v}, \bar{w} \in[v, w]$ and $\bar{v}^{\prime}, \bar{w}^{\prime} \in\left[v, w^{\prime}\right]$ whenever $\bar{v}, \bar{w} \in\left[v_{0}, w_{0}\right]$ are coupled quasisolutions of (4.1) such that $\bar{v}^{\prime}, \bar{w}^{\prime} \in\left[v_{0}^{\prime}, w_{0}^{\prime}\right]$.

Next we shall give some special cases of the above result. Given an ordered Banach 
space $E$ with order cone $K$, denote by $L(E)$ the Banach space of all bounded linear mappings $A: E \rightarrow E$ with the norm $\|A\|=\sup \{\|A x\|\|\| x \| \leq 1\}$. We say that $A \in L(E)$ is positive if $A[K] \subset K$. Denote by $I$ the identity mapping of $E$.

Lemma 4.2: The hypothesis ( $f a$ ) holds, for each choice of $u_{0_{i}}, u_{1_{i}} \in E$, $i=1, \ldots, n$, if for each $i=1, \ldots, n$ there exist $A_{i}^{j}, B_{i}^{j} \in L_{1}(J, L(E)), C_{i}^{j} \in L_{1}(J, E)$ and $h_{i}^{j} \in L_{1}(J, \mathbb{R}), j=1,2$ such that each $A_{i}^{j}(t)+h_{i}^{j}(t) I$ and $B_{i}^{j}(t)$ is positive for a.e. $t \in J$, and that

$$
A_{i}^{1}(t) v+B_{i}^{1}(t) u_{i}+C_{i}^{1}(t) \leq f_{i}(t, u, v) \leq A_{i}^{2}(t) v+B_{i}^{2}(t) u_{i}+C_{i}^{2}(t) \text { for a.e. } t \in J \text {. }
$$

Proof: Let $u_{0_{i}}, u_{1_{i}} \in E, i=1, \ldots, n$ be given. From Lemma 6.1 of [3] it follows that the IVP

$$
u^{\prime \prime}(t)=A_{i}^{j}(t) u^{\prime}(t)+B_{i}^{j}(t) u(t)+C_{i}^{j}(t) \text { for a.e. } t \in J, u(0)=u_{0_{i}}, u^{\prime}(0)=u_{1_{i}}
$$

has for each $i=1, \ldots, n$ the uniquely determined solution $u=v_{0_{i}}$ when $j=1$, and $u=w_{0_{i}}$ when $j=2$, and that $v_{0_{i}} \leq w_{0_{i}}$ and $v_{0_{i}}^{\prime} \leq w_{0_{i}}^{\prime}$ for each $i=1, \ldots, n$. From (4.6) it follows that $v_{0}=\left(v_{0_{1}}, \ldots, v_{0_{n}}\right)$ and $w_{0}=\left(w_{0_{1}}, \ldots, w_{0_{n}}\right)$ are coupled lower and upper quasisolutions of (4.1).

In the case when $p_{i}=n-1$ and $q_{i}=0$ for each $i=1, \ldots, n$ we obtain the following result concerning the existence of extremal solutions of the system

$$
\begin{gathered}
u_{i}^{\prime \prime}(t)=f_{i}\left(t, u(t), u_{i}^{\prime}(t)\right) \text { for a.e. } t \in J \\
u_{i}(0)=u_{0_{i}}, u_{i}^{\prime}(0)=u_{1_{i}}, i=1, \ldots, n .
\end{gathered}
$$

Proposition 4.2: Given an ordered Banach space $E$ with regular order cone and $f_{i}: J \times E^{n+1} \rightarrow E, \quad i=1, \ldots, n$, assume that the hypotheses of Lemma 4.2 hold, that $f(\cdot, u(\cdot), v(\cdot))$ is strongly measurable whenever $u^{\prime}$ and $v$ are absolutely continuous and a.e. differentiable on $J$, and that there is $\mu_{i} \in L_{1}\left(J, \mathbb{R}_{+}\right)$such that $(u, v) \mapsto f_{i}(t, u, v)+\mu_{i}(t) v$ is increasing for a.e. $t \in J$ and for each $i=1, \ldots, n$. Then for each choice of $u_{0_{i}}, u_{1_{i}} \in E$, $i=1, \ldots, n$ the system $(4.8)$ has solutions $v$ and $w$ such that $\left(v, v^{\prime}\right) \leq\left(u, u^{\prime}\right) \leq\left(w, w^{\prime}\right)$ for all solutions $u$ of (4.8).

Proof: Let $u_{0_{i}}, u_{1_{i}} \in E, i=1, \ldots, n$ be given. It is easy to show that the hypotheses of Theorem 4.1 hold with $v_{0}, w_{0}$ given as in the proof of Lemma 4.2. If $u=\left(u_{1}, \ldots, u_{n}\right)$ is any solution of (4.8), it follows from (4.6) that $u_{i}$ is for each $i=1, \ldots, n$ an upper solution of (4.7) when $j=1$, and a lower solution when $j=2$. This implies by Lemma 
6.1 of [3] that $\left(v_{0}, v_{0}^{\prime}\right) \leq\left(u, u^{\prime}\right) \leq\left(w_{0}, w_{0}^{\prime}\right)$. This holds for each solution $u$ of $(4.8)$, so that the assertions follow from the results of Theorem 4.1.

Remark 4.1: $\quad$ If $E$ is separable and each $f_{i}$ is standard then the condition $(f b)$ and the corresponding hypothesis of Proposition 4.2 hold (cf. [8]). These assumptions hold also without separability of $E$ in the special cases when each $f_{i}$ is a Carathéodory function, or all the functions in question are continuous. In these special cases we obtain analogous consequences for systems (4.1), (4.8) and (2) as stated in Remarks 3.1 for first order systems.

The regularity of the order cone $K$ of $E$ is essentially used in the proofs of Theorems 3.2 and 4.1. This holds if, for instance, $E$ is finite-dimensional, or $E$ is a real Hilbert space, and $(x \mid y) \geq 0$ for all $x, y \in K$, or $E$ is reflexive and $K$ is normal. In particular, the nonnegative elements form a regular cone in the $L^{p}$-spaces of real-valued functions, defined on any measured space $\Omega$, if $1 \leq p<\infty$. More generally, if $K$ is a regular order cone in $E$, then the cone $L^{p}(\Omega, K)$ of a.e. $K$-valued functions of $L^{p}(\Omega, E)$ is regular in $L^{p}(\Omega, E)$. The nonnegative sequences form a regular order cone in $l^{p}$-spaces with $1 \leq p<\infty$, and also in $c_{0}$.

The above examples of ordered Banach spaces with regular order cone imply that the results of Theorems 3.2 and 4.1 can be applied to finite and infinite systems of first and second order initial value problems, as well as to finite systems of first and second order stochastic initial value problems.

\section{REFERENCES}

[1] S. Heikkilä, "On differential equations in ordered Banach spaces with applications to differential systems and random equations", Differ. Integral Eq. 3, 3 (1990), pp. 589600 .

[2] S. Heikkilä, V. Lakshmikantham, and Y. Sun, "Fixed point results in ordered normed spaces with applications to abstract and differential equations", J. Math. Anal. Appl. (to appear).

[3] S. Heikkilä and S. Leela, "On solvability of second order initial value problems in Banach spaces”, manuscript (1991).

[4] E. Hille, “Methods in Classical and Functional Analysis”, Addison-Wesley, 1972.

[5] G.W. Howell and V. Lakshmikantham, "A new approach to monotone iterative technique for solution of differential equations", Appl. Anal. 39, 2-3 (1990), pp. 113-117.

[6] G.S. Ladde, V. Lakshmikantham and A.S. Vatsala, "Monotone Iterative Techniques for Nonlinear Differential Equations", Pitman, 1985.

[7] E.J. McShane, "Integration”, Princeton University Press, 1972. 
[8] I.V. Shragin, “On the Carathéodory conditions”, Russian Math. Surveys 34, 3 (1979), pp. 220-221.

[9] Y. Sun, "A fixed point theorem for mixed monotone operators with applications", $J$. Math. Anal. Appl. 156, (1991), pp. 240-252. 


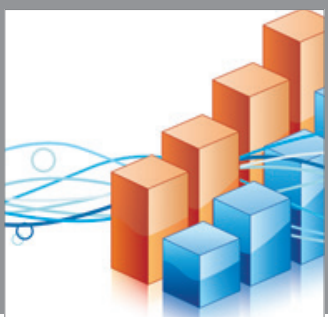

Advances in

Operations Research

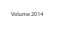

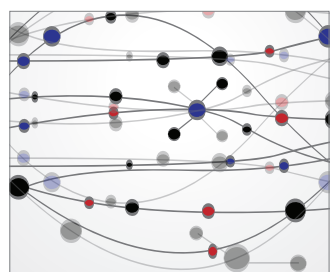

\section{The Scientific} World Journal
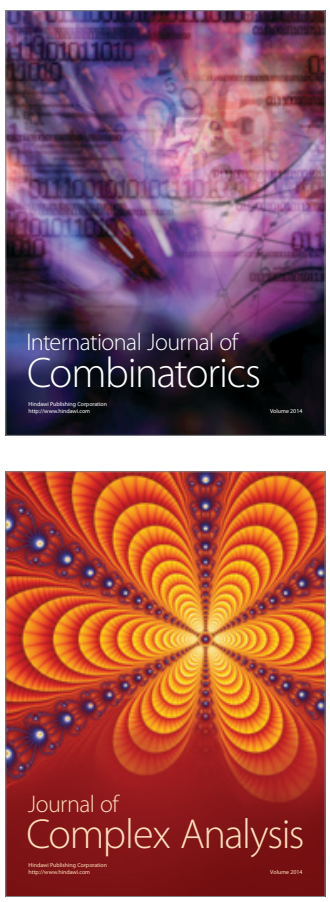

International Journal of

Mathematics and

Mathematical

Sciences
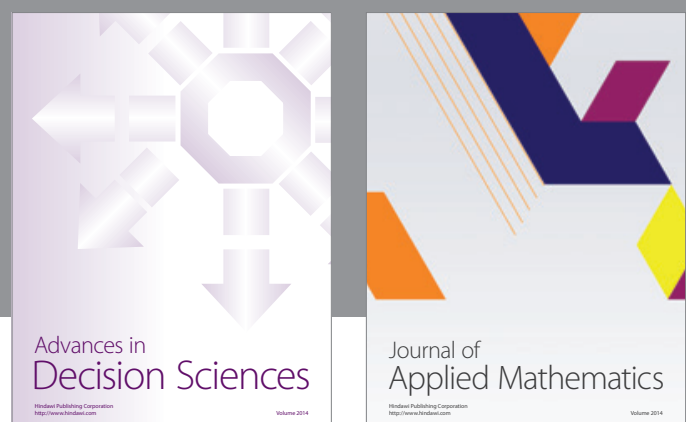

Journal of

Applied Mathematics
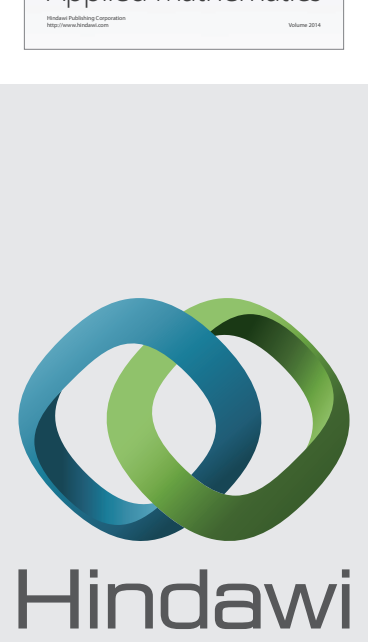

Submit your manuscripts at http://www.hindawi.com
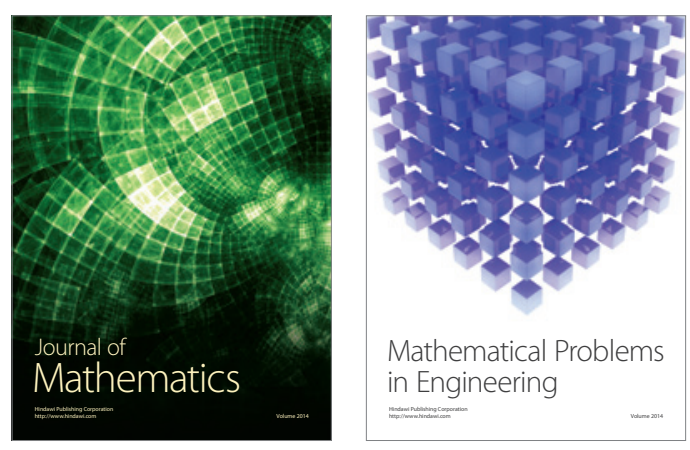

Mathematical Problems in Engineering
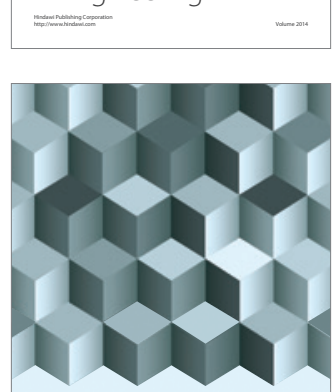

Journal of

Function Spaces
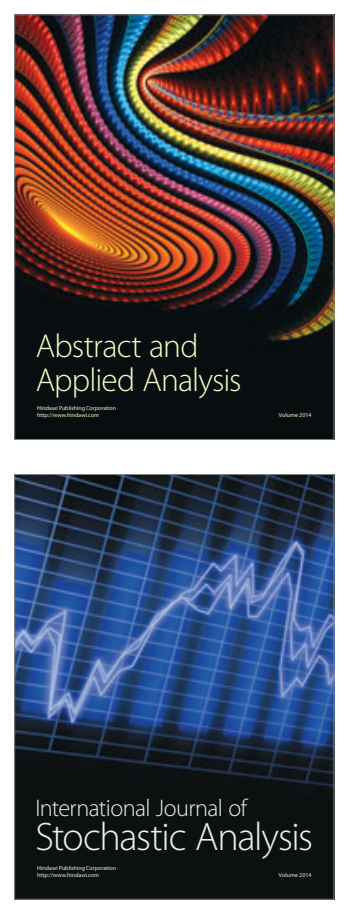

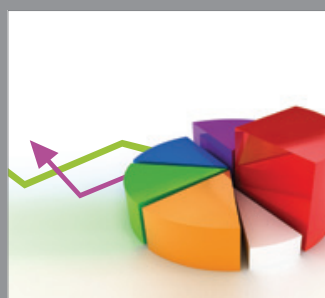

ournal of

Probability and Statistics

Promensencen
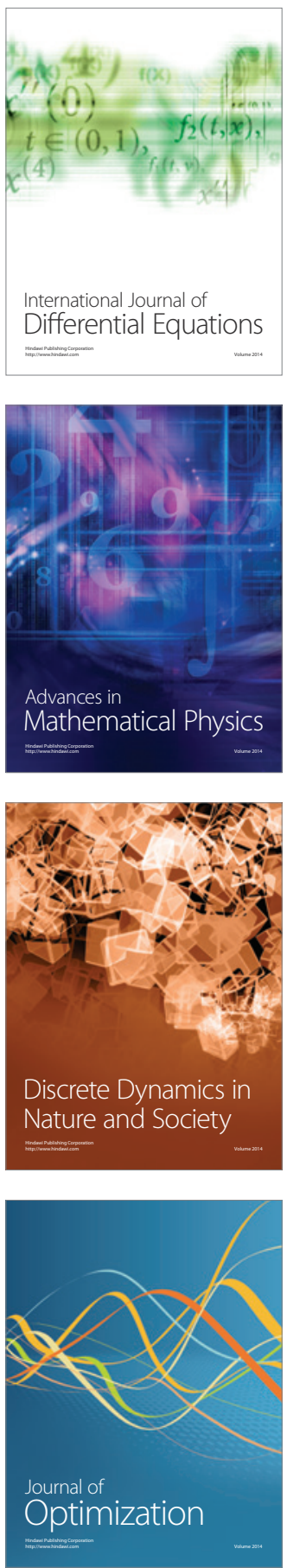